\title{
Optimal contact process on complex networks
}

\author{
Rui Yang, ${ }^{1}$ Tao Zhou, ${ }^{2,3}$ Yan-Bo Xie, ${ }^{2}$ Ying-Cheng Lai, ${ }^{1,4}$ and Bing-Hong Wang ${ }^{2}$ \\ ${ }^{1}$ Department of Electrical Engineering, Arizona State University, Tempe, Arizona 85287, USA \\ ${ }^{2}$ Department of Modern Physics, University of Science and Technology of China, Hefei 230026, China \\ ${ }^{3}$ Department of Physics, University of Fribourg, Chemin du Muse 3, CH-1700 Fribourg, Switzerland \\ ${ }^{4}$ Department of Physics, Arizona State University, Tempe, Arizona 85287, USA
}

\begin{abstract}
Contact processes on complex networks are a recent subject of study in nonequilibrium statistical physics and they are also important to applied fields such as epidemiology and computer and communication networks. A basic issue concerns finding an optimal strategy for spreading. We provide a universal strategy that, when a basic quantity in the contact process dynamics, the contact probability determined by a generic function of its degree $W(k)$, is chosen to be inversely proportional to the node degree, i.e., $W(k) \sim k^{-1}$, spreading can be maximized. Computation results on both model and real-world networks verify our theoretical prediction. Our result suggests the determining role played by small-degree nodes in optimizing spreading, in contrast to the intuition that hub nodes are important for spreading dynamics on complex networks.
\end{abstract}

DOI: 10.1103/PhysRevE.78.066109 PACS number(s): 89.75.Hc, 05.45.Xt, 05.70.Ln, 87.23.Ge

Phase transition with an absorbing state is a fundamental phenomenon in nonequilibrium statistical physics [1-3]. Such a transition can usually be described in terms of an order parameter, say $\rho$, as a function of some system parameter $p$. The absorbing state is defined by $\rho=0$, while $\rho>0$ corresponds to an active state. As $p$ changes through a critical point $p_{c}$, the order parameter changes from $\rho=0$ to $\rho$ $>0$, signifying a phase transition. The contact process (CP), introduced by Harris [4] as a model for epidemic spreading, has found wide applications in science and engineering [1,5]. Recently, the CP has been adopted to complex networks $[6,7]$ as a prototype model for investigating phase transition on large networks. A typical CP starts with a set of nodes that are infected by a virus or carry certain information. As time goes, these "seed" nodes interact with their neighbors so that the virus or information begins to spread on the network. The virus has a finite lifetime, which can be characterized by a probability $q$ that it can survive on a node for a basic time step determined by the specific physics of the problem. The parameter $p=1-q$ is thus the probability that the virus dies in a time step. If $p$ is sufficiently large, say $p>p_{c}$, the virus cannot spread and will vanish in a finite number of time steps, signifying an absorbing state. An active phase arises for $p<p_{c}$ where the virus can spread to a substantial part of the network. A key quantity in the CP dynamics is the contact probability, denoted by $W(k)$, which is the probability that a node of degree $k$ is infected upon being "contacted" by a neighboring node that has already been infected. In recent works [6,7], $W(k)$ is assumed to be degree independent, or uniform across the network. Under this assumption various scaling laws associated with the phase transition can be obtained and deviations from predictions of the mean-field theory have been addressed [6].

There are significant applications of CP on complex networks, such as wireless multihop ad hoc communication networks [8] and information infrastructure in defense networks, where efficient spreading of information is important. An issue thus concerns the optimal strategy for information spreading. Since network dynamics involve many quantities and parameters that can or cannot be controlled, in order to be able to address the "optimization" issue in a realistic way, we focus on the contact probability $W(k)$. In particular, suppose $p$ is fixed such that the network is in an active phase. We ask, what form of $W(k)$ should one choose so as to maximize the spreading? Let $\rho$ be the fraction of nodes in the network that are infected in a steady state. Our question is thus how to maximize $\rho$ by choosing $W(k)$. Since infinitely many possibilities for the functional form of $W(k)$ may exist, we shall further focus on the power-law function $W(k) \sim k^{\beta}$, where $\beta$ is a scaling exponent, as power law is one of the most commonly occurring scaling laws in nature, particularly associated with phase transitions. Our extensive simulation results using different complex-network topologies and configurations and real-world networks indicate that optimal spreading occurs for $W(k) \sim k^{-1}$. To explain this, we have developed a general theory, not constrained by any specific form of $W(k)$, which indeed predicts the optimal choice of $W(k) \sim k^{-1}$. For further verification, we examine, for the optimal choice of $W(k)$, the maximum value of $\rho$ as a function of the control parameter $p$. The theoretically predicted $\rho_{\text {max }}-\mathrm{vs}-p$ relation agrees reasonably well with the numerical results. A somewhat counterintuitive implication of our finding is that nodes with smaller degrees can play a significant role in information spreading. In particular, say, an infected node has a number of neighbors with different degrees and for a given time step it can only contact one neighboring node to spread the information. Our result indicates that the neighboring node with the smallest degree should be picked with higher probability, in order to maximize the final population of nodes that are infected with the information. Contacts with hub nodes, nodes with relatively large degrees in the network, should be depressed. Indeed, our theory suggests a destructive role played by the hub nodes in the $\mathrm{CP}$ based spreading dynamics. As many networked systems in nature and in various technological applications are complex with heterogeneous degree distributions, our result can be useful for a better understanding of epidemic dynamics and for designing networks that are most efficient for information spreading.

We consider a general CP process on complex networks. 


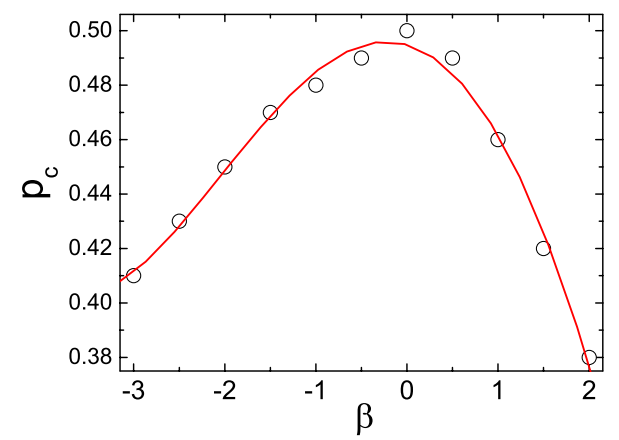

FIG. 1. (Color online) For a scale-free network of size $N$ $=2000$ and average degree $\langle k\rangle=6$, the critical probability $p_{c}$ vs the parameter $\beta$. Each data point is the result of averaging over $10^{4}$ runs of the $\mathrm{CP}$ process and each run contains $2 \times 10^{5}$ time steps, where a time average is taken for the last $10^{5}$ steps. The solid red line represents the polynomial fit of the data.

Assume that, initially, certain nodes in the network are infected (e.g., they catch a virus, or acquire a piece of information). For convenience, we say that a node, when infected, carries a particle. At each time step, an infected node is chosen at random. Say at a particular time step, node $i$ is chosen. The set of nodes that are connected to $i$ are its neighbors. With probability $p$, the particle carried by node $i$ dies, but with probability $1-p$, the particle can contact one of $i$ 's neighbors, say $j$. If $j$ is already occupied by a particle, it will not be affected. If, however, $j$ is not infected (or empty), the particle at node $i$ will generate an "offspring" on $j$, i.e., a new particle can be created on $j$. In existing works on $\mathrm{CP}$ on complex networks, the probability for a neighboring node to generate a particle is uniform among all the neighboring nodes of $i$. Here, we assume that the probability depends on the degree $k_{j}$ of the neighboring node $j$. As we have reasoned, this contact probability can be meaningfully chosen to be $W\left(k_{j}\right) \sim k_{j}^{\beta}$. Hence the probability of choosing node $j$ to accept the particle is

$$
\eta\left(k_{i}, k_{j}\right)=W\left(k_{j}\right) / \sum_{l \in V_{i}} W\left(k_{l}\right)=k_{j}^{\beta} / \sum_{l \in V_{i}} k_{l}^{\beta},
$$

where $l$ represents one of the nodes in the neighboring set of node $i, V_{i}$. If $\beta>0(\beta<0)$, a node with large (small) degree is more likely to be chosen. The fraction of infected nodes $\rho(t)$, which is equivalently the density of particles on the network, in general depends on time. If $p$ is large, there is a high likelihood that a particle will die before generating any offspring. In this case, the infection will eventually vanish and the asymptotic steady-state solution is $\rho=0$, which corresponds to an absorbing phase. If $p$ is small, we expect the infection to spread over the network and a nonzero asymptotic steady-state solution for $\rho$ can arise, signifying an active phase. As $p$ is increased from zero, there will be a phase transition to an absorbing state at $p_{c}>0$. For uniform contact probability, i.e., $\beta=0$, the critical value of $p_{c}$ is 0.5 [1]. For $\beta \neq 0$, the value of $p_{c}$ deviates from 0.5 .

To illustrate the deviation of $p_{c}$ from the idealized value 0.5 in the general case of nonuniform contact probability, we consider a standard scale-free network [9]. The number of
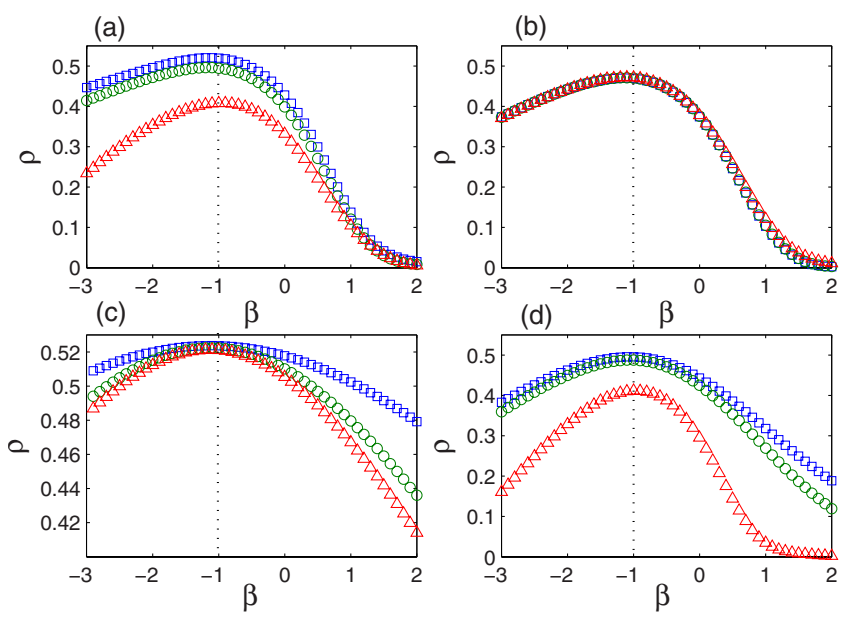

FIG. 2. (Color online) For $p=0.28$, steady-state particle density $\rho$ as a function of $\beta$ : (a) standard scale-free networks [9] of size $N=2000$ but with different average degrees, $\langle k\rangle=16$ (squares), 8 (circles), 4 (triangles); (b) scale-free networks of average degree $\langle k\rangle=6$ but with different sizes, $N=10000$ (squares), 5000 (circles), 1000 (triangles); (c) standard small-world networks [10] with different degrees of randomness as determined by the rewiring probability, $\phi=0.2$ (squares), 0.5 (circles), 0.8 (triangles); (d) scale-free networks of different degree exponent $\gamma=4$ (squares), 3.5 (circles), 2.5 (triangles) generated by the algorithm in [11]. In (c) and (d), the network size and the average degree are fixed as $N=2000$ and $\langle k\rangle$ $=6$. Every data point is the average result of $10^{4}$ independent runs of the $\mathrm{CP}$ dynamics. The remarkable phenomenon is that, regardless of the network topology and configuration, the maximum value of particle density is apparently achieved for $\beta=-1$.

nodes is $N=2000$ and the average degree is $\langle k\rangle=6$. We start the $\mathrm{CP}$ dynamics with the initial state where half of the nodes are infected. For a fixed value of $\beta$, the parameter $p$ is increased systematically from zero. For each value of $p$, the dynamics is evolved for a sufficiently long time to allow the system to settle down to a steady state, either absorbing or active. Thus the value of $p_{c}$ can be determined for each fixed value of $\beta$. The result is shown in Fig. 1, where we observe that for $\beta=0$, the value of $p_{c}$ is indeed 0.5 . When $\beta$ is not zero, we see that $p_{c}$ decreases from the idealized value 0.5 . This can be understood heuristically by taking into account the correlations in the dynamics. First consider the case where $\beta$ is positive. In this case, nodes of large degrees (hub nodes) are contacted more frequently than other nodes in the network. The particle density on the hub nodes is typically higher than the average density in the network. Thus only a small fraction of contacts, the contacts by the hub nodes, are likely to generate new particles. Many contacts in the network tend to generate no particles. In order for an average particle to survive on the network, the survival probability $q=1-p$ needs to be larger. This reduces the threshold probability $p_{c}$. Now consider the opposite case where $\beta$ is negative. In this case, nodes with relatively small degrees are contacted more often and these nodes tend to carry a relatively high density of particles. However, since these nodes are not well connected in the network, the number of contacts made by them is small. It is more difficult to generate particles at other nodes. In order to sustain a steady popula- 
tion of particles, the particle survival probability $q$ needs to be larger, effectively reducing the critical value $p_{c}$.

For the range of $\beta$ values considered in Fig. 1, the minimum value of $p_{c}$ is $p_{c}^{\text {min }} \approx 0.38$. To ensure that the network is in an active phase for all values of $\beta$ of interest, it is necessary to choose $p<p_{c}^{\min }$. In subsequent numerical experiments, we choose $p<0.38$.

To address the optimization issue, we compute, for a number of different network topologies and configurations, in the active phase, the steady-state particle density $\rho$ vs $\beta$. The results are summarized in Figs. 2(a)-2(d). Despite the different network topologies (scale-free and small-world of different degrees of randomness) and wide variations in network parameters such as the size, the average degree, and the degree exponent, we observe that $\rho$ is maximized for $\beta=-1$. Thus for the generic class of power-law, degree-dependent contact probability $W(k)$, the optimal strategy for spreading information most efficiently is achieved for $W(k) \sim k^{-1}$.

We now provide a mean-field theory to explain the optimal spreading strategy $W(k) \sim k^{-1}$. Let $n_{k}$ be the fraction of nodes of degree $k$ that are occupied by particles. The evolution of $n_{k}$, after incorporating the contact probability $W(k)$, can be written as

$$
\frac{\partial n_{k}}{\partial t}=-p n_{k}+(1-p)\left(1-n_{k}\right) k \sum_{k^{\prime}} \frac{P\left(k^{\prime} \mid k\right)}{k^{\prime}} \eta\left(k, k^{\prime}\right) n_{k^{\prime}},
$$

where the first term is due to the disappearance of particles with probability $p$, and the second term is due to the generation of new particles. Specifically, the fraction of empty nodes of degree $k$ is $\left(1-n_{k}\right)$. To generate a new particle at an empty node, it must be connected to an occupied node of some degree, say $k^{\prime}$, the probability of which is conditional and denoted by $P\left(k^{\prime} \mid k\right)$. The quantity $\eta\left(k, k^{\prime}\right)$ is the normalized contact probability given by

$$
\eta\left(k, k^{\prime}\right)=\frac{W(k)}{\sum_{k^{\prime \prime}} W\left(k^{\prime \prime}\right) P\left(k^{\prime \prime} \mid k^{\prime}\right)},
$$

where $k^{\prime \prime}$ denotes the degree of nodes in the neighbors of nodes of degree $k$. For an uncorrelated network, the conditional probability $P\left(k^{\prime} \mid k\right)$ is given by $P\left(k^{\prime} \mid k\right)=k^{\prime} P\left(k^{\prime}\right) /\langle k\rangle$ [12]. Substituting this into Eq. (2), we obtain

$$
\frac{\partial n_{k}}{\partial t}=-p n_{k}+(1-p)\left(1-n_{k}\right) k W(k) \frac{\rho}{\langle k W(k)\rangle},
$$

where $\rho=\Sigma_{k} P(k) n_{k}$ is the average density of particles, and $\langle f(k)\rangle \equiv \Sigma_{k} P(k) f(k)$ represents the expectation of some function of degree, say $f(k)$. The stationary solution to Eq. (4) is

$$
n_{k}=\frac{\lambda k W(k) \rho}{\langle k W(k)\rangle+\lambda k W(k) \rho},
$$

where $\lambda=(1-p) / p$. Carrying out the operation $\Sigma_{k} P(k)$ on both sides of Eq. (5), we obtain

$$
1=\sum_{k} P(k) \frac{\lambda k W(k)}{\langle k W(k)\rangle+\lambda k W(k) \rho}=\left\langle\frac{\lambda F(k)}{\langle F(k)\rangle+\lambda F(k) \rho}\right\rangle,
$$

where $F(k)$ denotes $k W(k)$. We see that, given a fixed value of $\lambda, \rho$ is a functional of $F(k)$. Taking the functional derivative $\delta / \delta F\left(k_{0}\right)$ with respect to Eq. (6), we get

$$
\begin{array}{r}
0=\left\langle\frac{\lambda \delta\left(k-k_{0}\right)(\langle F\rangle+\lambda F \rho)-\lambda F\left\{\left\langle\delta\left(k-k_{0}\right)\right\rangle+\lambda \rho \delta\left(k-k_{0}\right)+\lambda F\left[\delta \rho / \delta F\left(k_{0}\right)\right]\right\}}{(\langle F\rangle+\lambda F \rho)^{2}}\right\rangle \\
=\left\langle\frac{\lambda\langle F\rangle \delta\left(k-k_{0}\right)-\lambda F\left\langle\delta\left(k-k_{0}\right)\right\rangle-\lambda^{2} F^{2} \frac{\delta \rho}{\delta F\left(k_{0}\right)}}{(\langle F\rangle+\lambda F \rho)^{2}}\right\rangle
\end{array}
$$

and hence

$$
\frac{\delta \rho}{\delta F\left(k_{0}\right)}=\left\langle\frac{\langle F\rangle \delta\left(k-k_{0}\right)-F\left\langle\delta\left(k-k_{0}\right)\right\rangle}{(\langle F\rangle+\lambda F \rho)^{2}}\right\rangle /\left\langle\frac{\lambda F^{2}}{(\langle F\rangle+\lambda F \rho)^{2}}\right\rangle .
$$

The density $\rho$ can be maximized if $\delta \rho / \delta F\left(k_{0}\right)=0$. A possible solution is $\partial_{k} F(k)=0$, which leads to the optimal contact probability: $W(k) \sim k^{-1}$, in good agreement with results from numerical computations on model complex networks as in Fig. 2. Mathematically, there can be multiple choices of the function $F(k)$ to make the derivative in Eq. (9) vanish. Our choice $\partial_{k} F(k)=0$ is thus only suggestive and its validity needs to be checked by numerical computations. Indeed, as
Fig. 2 shows, extensive simulations on complex networks of different topologies for different parameter settings indicate that the choice is reasonable. It should be noted that this result is not valid for relatively large $p$. As shown in Fig. 1, for some $p$ (e.g., $p=0.49$ ), the system with $\beta=0$ is in the active state while the one with $\beta=-1$ is in the absorbing state. As a result, $\rho_{\beta=-1}$ is smaller than $\rho_{\beta=0}$, indicating the limitation of our result when $p$ approaches the critical value. 


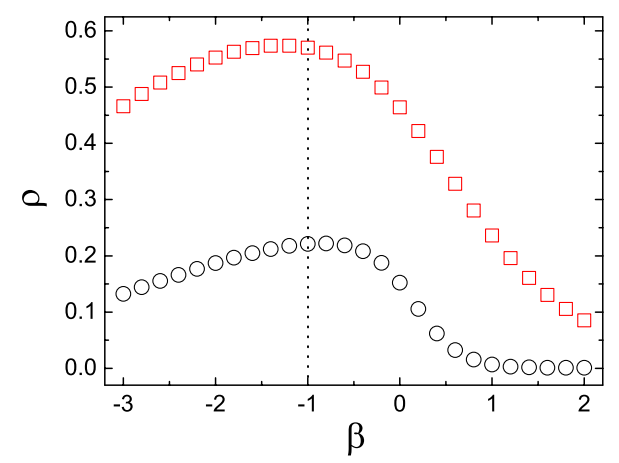

FIG. 3. (Color online) Contact process analysis (for $p=0.2$ ) for the Internet at autonomous system level (open circles) and for a co-author network (open squares) that includes all preprints posted between Jan 1, 1995 and March 31, 2005 [14]. The Internet has 22963 nodes and average degree $\langle k\rangle \approx 4.22$ and the co-author network has 40421 nodes and $\langle k\rangle \approx 8.69$. We see that the optimal spreading is achieved for $\beta \approx-1$.

To provide further credence, we have also performed computations using two real-world networks, the Internet and a co-author network, and have obtained consistent results, as shown in Fig. 3.

Note that, when the optimal functional form $W(k) \sim k^{-1}$ is substituted back into Eq. (6), we obtain $\rho_{\max }=1-1 / \lambda$ $=(1-2 p) /(1-p)$. Figure 4 shows, for a scale-free network, numerically obtained relations between $\rho_{\max }$ and $p$ (open circles) and the analytic prediction for $\rho_{\max }$ (open squares). We observe that, while there is a reasonable agreement between theory and numerics, the analytically predicted values of $\rho_{\max }$ are slightly larger than the corresponding numerical values. This can be explained as follows. Consider a particle located at node $i$, which is generated by another particle situated at one of $i$ 's neighboring nodes. Since this particle might have already generated some other particles in the neighborhood of $i$, the density of particles about $i$ can be larger than the asymptotic average density $\rho$. This means that, when two connected nodes in the same neighborhood are both occupied, the local density would be larger than $\rho$. Accordingly, in the simulation of the actual process, more contacts yield no new particles (or they are wasted), as compared with those in the theoretical process where the correlation effect is ignored. This leads to an overall lower value of $\rho$ in the simulation. This positive correlation effect becomes more significant for $p \rightarrow p_{c}$, leading to a non-mean-field type of behavior. These results are thus consistent with the main point of Ref. [6], where the failure of the mean-field approximation is systematically addressed for $p \rightarrow p_{c}$. A similar phenomenon is observed in a two-state epidemic spreading model where at least one of the neighbors of an infected node is infected [13]. In that case, we can replace the degree $k$ by the so-called remain degree $(k-1)$ [13]. However, the $\mathrm{CP}$ dynamics treated here is more complicated since there is a nonzero probability that the ancestor of a particle can disappear upon its generation.

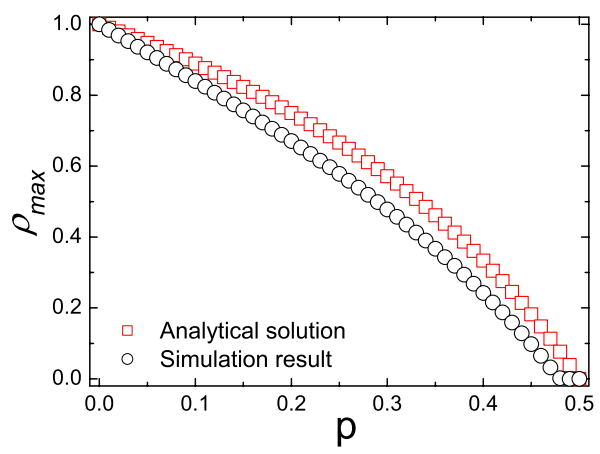

FIG. 4. (Color online) For a standard scale-free network of parameters $N=2000$ and $\langle k\rangle=6, \rho_{\max }$ (obtained with $\beta=-1$ ) vs the parameter $p$. Each numerical data point is the result of averaging over $10^{4}$ independent runs of $\mathrm{CP}$ dynamics.

In summary, we have found a universal strategy for the contact process on complex networks for optimal spreading under the condition that, for an infected node, only the local information about the degrees of its neighboring nodes is available. Insofar as the degree distribution is not uniform, we expect our strategy to be effective in maximizing the extent to which an infection (or a piece of information) can be spread on the network. Surprisingly, our optimal strategy emphasizes the role of small-degree nodes played in promoting efficient spreading dynamics. Moreover, recent studies on network-based traffic [16] have revealed that, when routers have a uniform delivering capacity, routing protocols preferring small-degree nodes outperform the traditional method without preference, and the optimal strategy is achieved in the case where the delivering probability is inversely proportional to the neighboring degree. Another example arises in coupled oscillators where the highest synchronizability can be achieved by setting the coupling strength inversely proportional to the degree [17]. Our result, besides its implications to the fundamentals of nonequilibrium statistical physics, potentially can have significant applications such as the design of broadcasting protocol of distributed sensor networks $[8,15]$. For a sensor network, the energy supply to each sensor is limited but the transmission of information costs a certain amount of energy. Carefully choosing the message receiver to avoid a waste of energy is of paramount interest. Our strategy also suggests that, in a social or a political network where the $\mathrm{CP}$ dynamics is relevant, seemingly unimportant nodes, i.e., nodes of relatively small degrees, can play a counterintuitively significant role in maximizing the impact of a certain message that the social or political leaders wish to spread.

We thank J. Ren, L. Huang, and Y. Wang for useful discussions. R.Y. and Y.C.L. are supported by AFOSR under Grant No. FA9550-07-1-0045. T.Z., Y.B.X. and B.H.W. acknowledge the support of China 973 Project No. 2006CB705500 and NSF of China under Grants No. 10635040 and No. 10472116. 
[1] J. Marro and R. Dickman, Nonequilibrium Phase Transitions in Lattice Models (Cambridge University Press, Cambridge, England, 1999).

[2] H. Hinrichsen, Adv. Phys. 49, 815 (2000).

[3] G. Ódor, Rev. Mod. Phys. 76, 663 (2004).

[4] T. E. Harris, Ann. Probab. 2, 969 (1974).

[5] R. Durrett, SIAM Rev. 41, 677 (1999).

[6] C. Castellano and R. Pastor-Satorras, Phys. Rev. Lett. 96, 038701 (2006).

[7] T. Zhou, J.-G. Liu, W.-J. Bai, G. R. Chen, and B.-H. Wang, Phys. Rev. E 74, 056109 (2006); R. Yang, B.-H. Wang, J. Ren, W.-J. Ren, Z. W. Shi, W.-X. Wang, and T. Zhou, Phys. Lett. A 364, 189 (2007).

[8] P. Gupta and P. R. Kumar, IEEE Trans. Inf. Theory 46, 388 (2000).

[9] A.-L. Barabási and R. Albert, Science 286, 509 (1999).

[10] D. J. Watts and S. H. Strogatz, Nature (London) 393, 440
(1998).

[11] M. E. J. Newman, S. H. Strogatz, and D. J. Watts, Phys. Rev. E 64, 026118 (2001).

[12] M. Boguñá, R. Pastor-Satorras, and A. Vespignani, Lect. Notes Phys. 625, 127 (2003).

[13] M. Barthélemy, A. Barrat, R. Pastor-Satorras, and A. Vespignani, Phys. Rev. Lett. 92, 178701 (2004).

[14] Both Internet and co-author network data are from http://wwwpersonal.umich.edu/mejn/netdata/

[15] I. F. Akyildiz, S. Weilian, Y. Sankarasubramaniam, and E. Cayirci, IEEE Commun. Mag. 40(8), 102 (2002).

[16] W.-X. Wang, B.-H. Wang, C.-Y. Yin, Y.-B. Xie, and T. Zhou, Phys. Rev. E 73, 026111 (2006); G. Yan, T. Zhou, B. Hu, Z.-Q. Fu, and B.-H. Wang, ibid. 73, 046108 (2006).

[17] A. E. Motter, C. Zhou, and J. Kurths, Phys. Rev. E 71, 016116 (2005). 